Entre Ciencia e Ingeniería, vol.14, no. 28, Editorial, páginas 7-9. ISSN 1909-8367 (Impreso), ISSN 2539-4169 (En línea)

\title{
Editorial
}

\section{Entre Ingeniería, Tecnología y Productividad}

\section{Between Engineering, Technology and Productivity}

\author{
Lloyd Herbert Morris Molina, PhD.
}

La alternativa para sustentar la competitividad en las organizaciones es a través de la productividad. Este concepto relaciona la producción de bienes y servicios con respecto a los recursos utilizados como: mano de obra, materiales, energía, maquinaria u otros factores. En este sentido, una de las áreas del conocimiento que posee un rol protagonista para el incremento de la productividad es la ingeniería que, en función de un interesante trabajo relacionado con la comprensión del conocimiento, las habilidades, las competencias y el proceso de aprendizaje de índole ingenieril, logra el dominio de técnicas y de la tecnología en sus diversas etapas de innovación y desarrollo [1]. Por tal razón, la necesidad de optimizar los ciclos en los procesos de manufactura y de servicios, conllevan a que los esfuerzos de ingeniería utilicen modelos basados en la definición de capacidades en relación con el impacto en la productividad, como ejemplo de ello, se tienen los modelos en ciclos de manufactura con aplicaciones informáticas: CAD (Computer aided desing), CAE (Computer aided engineering), CAPP (Computer aided process planing) y CAM (Computer aided manufacturing) [2]. Ahora bien, la incorporación de modelos o técnicas deben tener una orientación hacia el cumplimiento de los objetivos en operaciones (calidad, flexibilidad, costos y tiempos de entrega), de tal manera que exista un enlace hacia su inserción en las estrategias de fabricación en industrias 4.0, para el incremento de la productividad. [3].

Los objetivos en operaciones están interrelacionados con aplicaciones tecnológicas, por ejemplo, la posibilidad de incrementar la flexibilidad y productividad en las redes de producción de la Industria 4.0, se puede alcanzar mediante la incorporación de robots autónomos móviles y con la intralogística inteligente [4], en cuanto a la calidad, por ejemplo, el incorporar metas de cero defectos, aumenta la productividad en procesos de las compañías de manufactura y servicios, utilizando seven QC (Quality Control) tools: Flow chart, check sheets, histogramas, Pareto diagram, cause and effect diagram, scatter diagram and p-chart en la integración de la información en plataformas computacionales [5]. Por tanto, se obtiene una mayor habilidad de reacción (flexibilidad en la oferta), con tiempos cortos de respuesta hacia los cambios en la demanda y la minimización de costes de producción en paralelo a mejores estándares de calidad que finalmente impactan de forma positiva en la productividad del sistema de producción.

El enfoque hacia la productividad desde la ingeniería posee su punto de apoyo en la tecnología, lo que ha permitido distintos frameworks basados en el ciclo de mejoramiento continuo: Check - Action - Plan - Do, con direccionamiento a procesos de rediseño y reingeniería. Precisamente, dentro de estos frameworks, se localiza el BPR (Business Process Re-engineering or Business Process Re-desingning), el cual posee una dirección al mejoramiento en ingeniería de procesos con relación a la 
productividad, calidad del producto y ventajas competitivas. [6,7]. Otro framework, que se puede considerar es el marco para el mejoramiento de la productividad que incorpora una orientación de las operaciones para el logro de metas de productividad agrupando cuatro áreas estratégicas o 4Ps (people, product, process and policy) [8,9]. Ahora bien, en el desarrollo comparativo entre los modelos citados BPR y 4Ps, se encuentra una estrecha relación en el requerimiento de iniciativas tácticas con bases metodológicas y tecnológicas para alcanzar la ingeniería de procesos mediante: Total quality management, Just intime, 5S, Kaizen, 3Rs (reduce, reuse and recycle), benchmarking, six - sigma, ISO, OHSAS y balance scorecard.

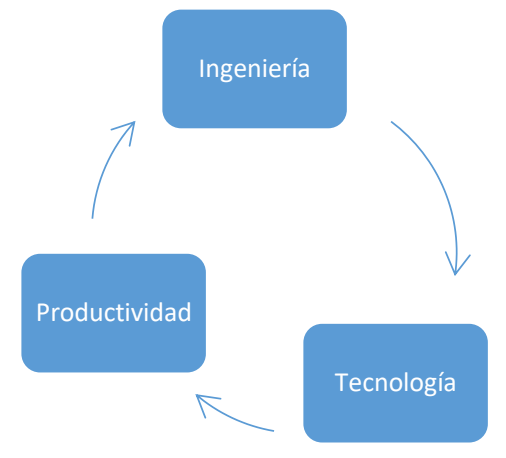

Fig. 1. Ingeniería, tecnología y productividad.

Por las razones expuestas, se puede deducir la necesaria y estrecha relación entre los conceptos bases de ingeniería más la incorporación de las técnicas, métodos y tecnologías para el alcance en procesos cíclicos y dinámicos de mejores niveles de productividad. En la fig. 1, se puede realizar la interpretación de las tres variables analizadas: los conocimientos, habilidades y competencias propias del ámbito de la ingeniería para el abordaje de problemas típicos de índole ingenieril, y con la incorporación de técnicas, herramientas y tecnologías para la optimización de los procesos en los que se logran alcanzar objetivos propios de las operaciones como: minimización de costos, flexibilización de la oferta, mejoramiento de la calidad y la reducción de tiempos para el incremento de la productividad organizacional.

\section{REFERENCIAS}

[1] Ronghuai Huang, J. Michael Spector and Junfeng Yang, Educational Technology, A Primer for the $21^{\text {st }}$ Century. Springer, Lecture notes in Educational Technology, 2019. DOI:10.1007/978-981-13-6643-7.

[2] Emmanouil Varitis, Konstantinos Rinos and Nikolaos Kostis, Model - based definition capabilities and its impact on industrial productivity, MATEC Web of Conferences 318, 01048 (2020), ICMMEN, 2020. DOI: https://doi.org/10.1051matecconf/202031801048.

[3] Mohammad Asif Salaf, King Abdulaziz, Analyzing manufacturing strategies and Industry 4.0 supplier performance relationship from a resource based perspectivie, Benchmarking: An International Journal. Emerald Publishing Limited, 2019. DOI:10.1108/BIJ-12-2018-0428.

[4] Giuseppe Fragapane, Dmitry Ivanov, Mirco Peron, Fabio Sgarbossa and Jan Ola Strandhagen, Increasing flexibility and productivity in Industy 4.0 production networks with autonomus mobile robots and smart intralogistics. Annals of Operations Research, Springer, February 2020 https://doi.org/10.1007/s10479-020-03526-7

[5] Imdad Ali Memon, Qadir Bakhsh Jamali, Abdul Sattar Jamali, Muhammad Kashif Abbasi, Nisar Almed Jamali and Zahid Hussain Jamali, Defect reduction with the use of seven quality control tools for productivity improvement at an automobile company. Engineering technology \& applied sacience research, Vol. 9, No 2, 2019, 4044-4047.

[6] Krumal J. Bhavsar, Vrutik Shah and Samir Gopalan, Process life cycle framework: A conceptual model and literature study of business process reengineering for software engineering management. CiiT International Journal of Software Engineering and Technology, Vol 11, No 6, June 2019. ISSN 0974-9748.

[7] Moayyad Al-Fawaeer, Mahmood B. Ridha and Abdul Sattar H. Yousif, An investigation into the relationship between business processes reengineering (BPR) and employees' performance: An empirical study at the Jordanian public shareholding companies. Review of Applied Socio Economic Research, Volume 17, Issue 1/2019. ISSN 2247-6172.

[8] Antonio D. Kalaw and Jr. Philippines, Handbook on Productivity. 50 years of the Asian Productivity Organization. First published in Japan 2015.

[9] Tom Gilb, Competitive Engineering: A handbook for systems engineering, requirements engineering, and software engineering, using planguage.Middiesex University, UK. 2005. 


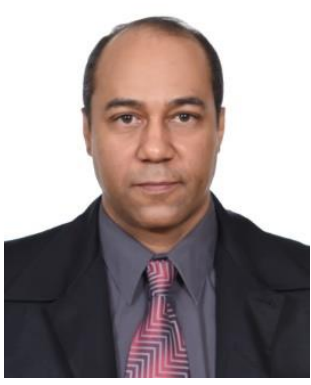

Lloyd Herbert Morris Molina es profesor en los programas de Ingeniería Industrial de la Universidad Tecnológica de Pereira y la Universidad Católica de Pereira - Colombia. Tiene títulos de ingeniería y maestría de la Universidad Nacional Experimental del Táchira en Venezuela, una maestría de la Universidad de Alcalá en España y un doctorado en Ciencias Gerenciales de la Universidad de las Fuerzas Armadas. Antes de venir a Colombia, fue profesor de Ingeniería Industrial en la Universidad Nacional Experimental del Táchira en Venezuela, donde impartió cursos de Producción. Además, ocupó diferentes cargos administrativos como Jefe de Tesorería, Planificador del Consejo de Planeación, Especialista Organizacional, director del Máster en Administración de Empresas, presidente de la Caja de Ahorros de la UNET y Director de la Escuela de Ciencias Administrativas (CIAF). Morris tiene 20 años de experiencia en educación superior en Colombia y Venezuela. A nivel de posgrado, ha sido profesor invitado en los programas de Administración de Empresas (ULA), Logística (UNEFA) y Gerencia de Empresas (UNET). Además, el Dr. Morris tiene varios años de experiencia en la industria de la Ingeniería de Fabricación, por ejemplo, en LAFARGE como Jefe de Fabricación. Finalmente, pertenece al grupo de investigación Entre Ciencia e Ingeniería, donde el objetivo del Dr. Morris es mejorar los procesos en las operaciones de Ingeniería a través de enfoques gerenciales que incorporan técnicas o herramientas matemáticas en los procesos para la toma de decisiones en pro de la productividad y competitividad. 